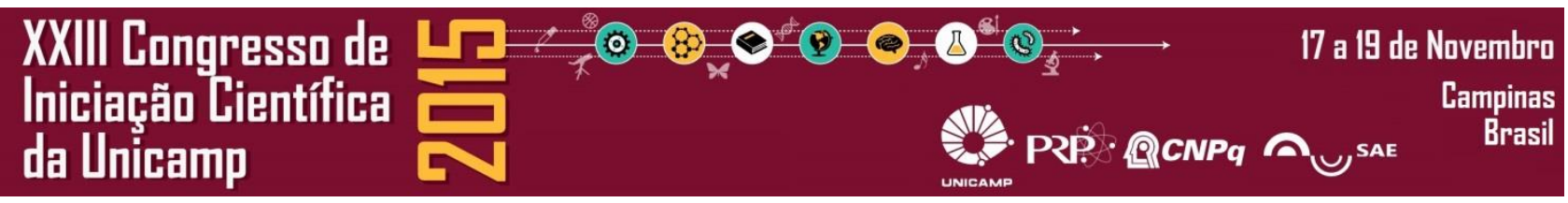

\title{
Third-harmonic generation in silicon oxide microcavities
}

Jorge H. Soares (IC), Felipe G. Santos (PG), Laís Fujii (IC),

Gustavo S. Wiederhecker (PQ) and Thiago P. Mayer Alegre (PQ).

\section{Abstract}

Third-harmonic generation (THG) is observed in wet-etched optical microcavities formed from silicon oxide. The THG spectra reveals an intensity enhancement due to the combination of the phase matching and fundamental light localization provided by the optical microcavities. Our device design along with a very effecttive collection of the visible lights through a optical tapered fiber allows for a net efficiency as high as $10^{-5}$.

Keywords: Non-linear optics, third harmonic generation, optical cavities.

\section{Introduction}

Optical microcavities are well known to provide high optical confinement and quality factors. New micro-fabrication techniques ${ }^{[1]}$ have driven such systems to a new frontier, where photon lifetime exceeds a microsecond. This allows for a large intracavity photon number enabling non-linear optics at moderate pump powers. Here we demonstrate an optical microcavity based upon a silicon-oxide substrate with optical quality factor as high as $10^{6}$ in the infrared region. These cavities where then used to produce thirdharmonic-generation (THG) which results in a highly efficient emission of visible light.

\section{Results and discussions}

THG is a non-linear process where the resulting optical frequency is three times that of the input laser beam. In silica microcavities this is achieved with a $x^{(3)}$ nonlinearity where the energy conservation imposes that three infrared photons (1545 nm) are combining into a single visible photon $(515 \mathrm{~nm})$. Such process is also bounded to momentum conservation (phase-matching) ensured by the different optical dispersion for optical modes in the infrared and visible region.

Our optical cavities were fabricated through a wet-etch ${ }^{2}$ process. The devices are defined by photolithography process and them etched using buffered hydrofluoric acid. Finally the silicon wafer is etched to form a pedestal using a potassium hydroxide solution. The fabrication results can be seen in Fig. $1 \mathrm{c}$ ).

Light is coupled into and out of the device using a specially prepared optical fiber taper ${ }^{[3]}$, which when placed in the near-field of the microdisk couples the guided modes of the taper evanescently to the optical resonances of the microcavity. An infra-red tunable laser is modulated and then amplified by an Erbiumoptical amplifier increasing the input peak power to few Watts. As a result the optical transmission exhibits a distinct thermal bistability behavior characterized by the sharp transmission curves at high input power as shown in Fig. 1 b).

Finally the infra-red (IR) and visible light (VIS) are separated by a prims and measured with two independent detectors. For the highest optical input power the generated visible light is intense enough to produce the glowing micrograph picture seen in Fig. 1d). By placing the optical fiber taper in different positions we could enhance the optical emission and collection efficiency of the visible light. As a result the maximum net efficiency defined as the ratio between the IR input power and output VIS light $\eta=P_{\mathrm{IR}} / P_{\mathrm{VIS}}$ exceeds $10^{-5}$, one of the largest ratio for such devices ${ }^{[4]}$.

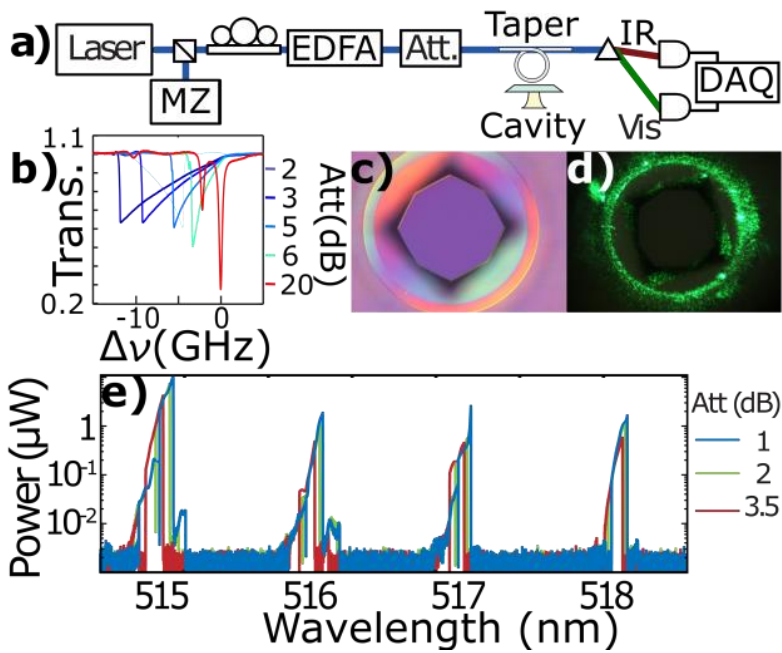

Figure 1. a) Experimental setup: Laser light source; MZ: Mach-Zehnder interferometer for optical calibration; Erbium doped fiber amplifier (EDFA) Vis and IR: visible and infrared optical detectors; DAQ: digital analog converter. b) Optical microscopy image of the fabricated sample. c) Microscope image of visible (green) light generated trough THG non-linear process. d) Optical transmission as a function of the input. e) Green light emission as a function of pump power.

\section{Conclusion}

We fabricated and characterized optical cavities, which presents high enough $Q$ factor to enable the now-linear effect such as THG at moderate input powers. The net efficiency of collection of the visible lights was high as $10^{-5}$.

\section{Acknowledgement}

We acknowledge CNPq (550504/2012-5) and FAPESP (2012/17610-3, 2012/17765-7 and 2013/06360-9) for financial support.

[1] K. J. Vahala, "Optical microcavities," Nature, 424, 839 (2003).

[2] Lee, H.; et al. Nature Photonics, 6, 369 (2012).

[3] Michael, et al. Opt. Express 15, 4745 (2007).

[4] Farnesi, D. Phys. Rev. Lett. 112, 093901 (2014). 